
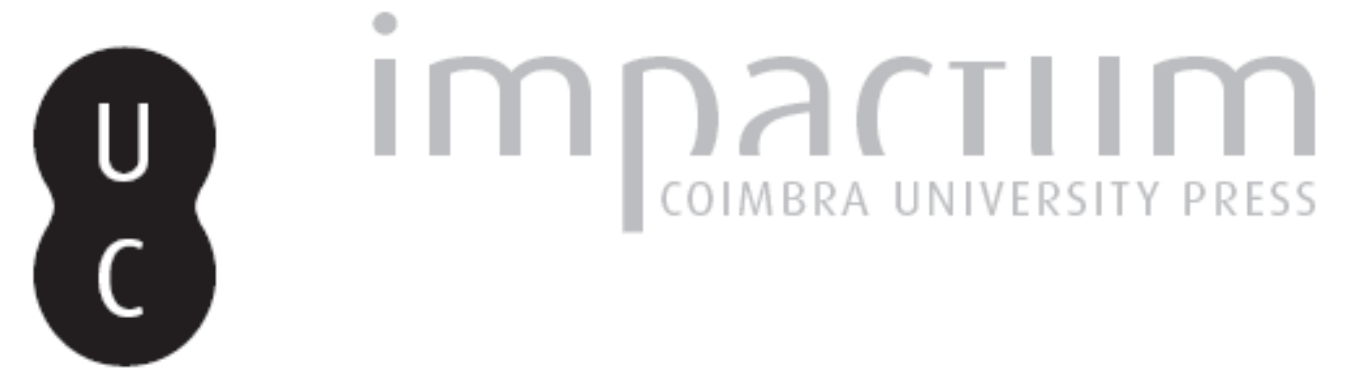

\title{
La critique augustinienne des catégories d' Aristote
}
Autor(es):
Villevieille, Laurent
Publicado por:
Faculdade de Letras da Universidade de Coimbra, Instituto de Estudos Filosóficos

URL

persistente:

DOI:

URI:http://hdl.handle.net/10316.2/33427

Accessed : $\quad$ 26-Apr-2023 08:31:04

DOI:http://dx.doi.org/10.14195/0872-0851_43_5

A navegação consulta e descarregamento dos títulos inseridos nas Bibliotecas Digitais UC Digitalis, UC Pombalina e UC Impactum, pressupõem a aceitação plena e sem reservas dos Termos e Condições de Uso destas Bibliotecas Digitais, disponíveis em https://digitalis.uc.pt/pt-pt/termos.

Conforme exposto nos referidos Termos e Condições de Uso, o descarregamento de títulos de acesso restrito requer uma licença válida de autorização devendo o utilizador aceder ao(s) documento(s) a partir de um endereço de IP da instituição detentora da supramencionada licença.

Ao utilizador é apenas permitido o descarregamento para uso pessoal, pelo que o emprego do(s) título(s) descarregado(s) para outro fim, designadamente comercial, carece de autorização do respetivo autor ou editor da obra.

Na medida em que todas as obras da UC Digitalis se encontram protegidas pelo Código do Direito de Autor e Direitos Conexos e demais legislação aplicável, toda a cópia, parcial ou total, deste documento, nos casos em que é legalmente admitida, deverá conter ou fazer-se acompanhar por este aviso.

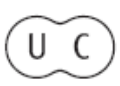




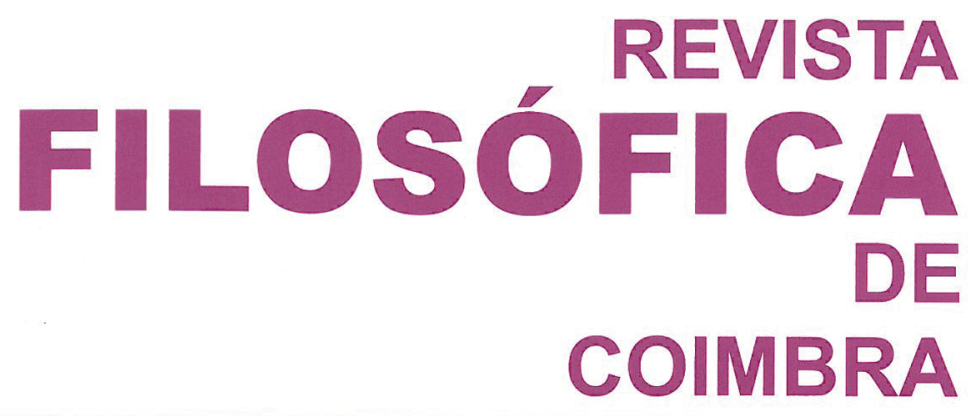

vol. 22 - número 43 - março 2013

vol. 22 - número 43 - março 2013

Fundação Eng. António de Almeida

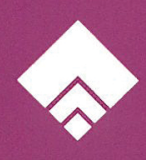




\title{
LA CRITIQUE AUGUSTINIENNE DES CATÉGORIES D'ARISTOTE
}

\author{
LAURENT VILLEVIEILLE*
}

Résumé: Dans le De Trinitate, Augustin ne critique les catégories d'Aristote que sur la base de leur réinterprétation hellénistique (d'origine néoplatonicienne et stoïcienne). La superficialité de cette critique permet cependant, contre toute attente, un renouvellement de la source même de ce qu'elle critique, et qu'elle méconnaît paradoxalement : le traité des Catégories d'Aristote. Ce qu'Augustin substitue alors à la parole apophantique qui en constitue le fond est moins une parole apophatique, que la découverte d'une propriété apophatique de la parole elle-même.

Mots-clefs: Augustin, Aristote, catégories, logique, substance, relation, apophantique, apophatique

Resumo: No De Trinitate Agostinho critica as categorias de Aristóteles na base da sua reinterpretação helenística (originalmente neoplatónica e estóica). A superficialidade da sua crítica permite, no entanto, e contra todas as expectativas, a renovação da própria fonte criticada, e que paradoxalmente não conhecia: o tratado das Categorias de Aristóteles. O que Agostinho substitui então à palavra apofântica que é o seu fundo não é tanto uma palavra apofática quanto a descoberta de uma propriedade apofática da própria palavra.

Palavras-chave: Agostinho, Aristóteles, categorias, lógica, substância, relação, apofântica, apofático

La phénoménologie ${ }^{1}$ permet singulièrement de relire l'histoire de la

* Université Paris-Sorbonne (Paris IV).

${ }^{1}$ La présente étude avait été présentée initialement dans le cadre du séminaire de Jean-Luc Marion consacré à la pensée de saint Augustin durant l'année universitaire 2006-2007. Nous tenons ici à remercier cordialement Jean-Luc Marion de la possibilité qu'il nous avait ainsi offerte d'exposer les grandes lignes de notre interprétation de la question 
philosophie comme une histoire du logos. C'est-à-dire non pas de « la parole ", qui en elle-même peut faire l'objet de toutes sortes d'approches - linguistiques, philologiques, grammaticales ou stylistiques. Mais de la parole en tant qu'elle ouvre au jour à la fois singulier et familier du phénomène tel qu'il se donne à entendre et à voir - ou plus justement : à voir à la faveur d'un entendre. Le logos, qui est en un sens cette faveur même, donne alors à la parole une dimension que son découpage disciplinaire en régions ne saurait atteindre : dimension sans laquelle tout découpage, toute région, ne pourraient pas même exister, puisqu'elle est au fond l'ouverture, initiale autant que décisive, de toute dimension possible. La philosophie apparaît alors comme tentative d'assomption d'une telle dimension, tentative qui a, elle peut-être plus que tout autre, son histoire.

$\mathrm{Au}$ nombre des chapitres importants de cette histoire figure en bonne place la question des catégories, soit des chefs d'accusation par lesquels l'étant est dit être tel ou tel. Une telle mise en accusation, longtemps prise comme fil directeur privilégié, pour ne pas dire exclusif, dans l'approche du logos, est cependant signalée, dès sa première prise en vue thématique, comme l'un seulement des modes du logos, dont la prière, par exemple, constitue, nous dit Aristote, une tout autre guise. ${ }^{2}$

De la réinterprétation possible de l'histoire de la philosophie comme histoire du logos, l'étude de la critique augustinienne des catégories d'Aristote est plus qu'un simple exemple. Saint Augustin, loin de chercher à renouveler le logos catégorial, dénoncé insuffisant, dans une autre forme de logos - par exemple la prière, justement ${ }^{3}$ - parvient à renouveler un tel logos depuis son intérieur même.

Dans les livres V à VII du De Trinitate, saint Augustin élabore en effet ce que nous appellerons provisoirement une logique trinitaire. Celle-ci est, à différents égards, une révolution. Pour n'en citer que l'apport le plus manifeste, cette logique parvient à renouveler la théorie de la prédication. Comme nous le verrons plus loin, la prédication est traditionnellement conçue comme soit substantielle («Callias est un homme »), soit accidentelle («Callias est blanc»). En deçà de cette alternative, saint Augustin

catégoriale dans la pensée de saint Augustin, interprétation qui doit tant à ses propres travaux, lesquels préparaient encore, à l'époque, la publication de son livre Au lieu de soi. L'approche de Saint Augustin, Paris, P.U.F. (coll. «Epiméthée »), 2008.

2 Aristote, De l'interprétation, IV, 17a5 (pour le texte grec, nous suivons l'édition anglaise de H. P. Cook et H. Tredennick, Aristotle. Categories. On interpretation. Prior Analytics, Cambridge, Loeb Classical Library, 1996²).

${ }^{3}$ Saint Augustin a aussi été l'auteur d'un tel renouvellement qui, loin de se limiter à la prière comme à un acte unique et unitaire, se décline dans toutes les modalités de la parole que Jean-Louis Chrétien a si remarquablement élucidées dans son ouvrage Saint Augustin et les actes de parole, Paris, P.U.F. (coll. " Epiméthée »), 2002. 
découvre un tiers jusqu'alors exclu : la prédication selon la relation. Ce renouvellement de la catégorie de la relation, qui assure à la parole qui tente de nommer la Trinité un discours moins inadéquat que le discours traditionnel, constitue un apport décisif pour le discours théologique. Mais cet apport dépend plus profondément d'une révolution du discours en tant que tel, c'est-à-dire d'une révolution logique. Cependant, cette révolution en est-elle bien une ? Autrement dit, est-elle bien un revolvere - un retour à la source de ce qu'elle révolutionne?

Le paradoxe est ici que ce retour à la source semble se faire à l'insu même de celui qui l'opère. Comme nous n'allons pas tarder à le voir, la critique augustinienne des catégories se fonde sur une lecture hellénistique de ce qui, au fil des siècles, a fini par devenir une tradition et, pour tout dire, un quasi bien connu de la philosophie antique tardive. Mais tout en se fondant sur cette interprétation superficielle des catégories, la critique augustinienne parvient, contre toute attente, à renouveler la source même de ce qu'elle critique, et que paradoxalement elle méconnaît pourtant, à savoir les Catégories d'Aristote.

La thèse que nous voudrions alors défendre est celle d'une révolution, dans la pensée augustinienne de la Trinité, non seulement de l'aristotélisme, mais bien de la pensée d'Aristote. Plus profondément que la tradition logique, ce que la critique augustinienne parviendrait à atteindre, fût-ce à son insu, serait ainsi la source même de cette tradition. Loin de n'être que l'un des chapitres, fût-il éminent, de l'histoire de la logique, elle participerait à l'ouverture d'une nouvelle dimension du logos. Hypothèse de lecture qui implique au moins deux choses.

Tout d'abord de distinguer la pensée d'Aristote de l'aristotélisme hellénistique, avec lequel seulement est en débat saint Augustin. Donc de libérer la pensée d'Aristote des interprétations qui en ont été proposé dans l'Antiquité tardive. Non pas pour prétendre exposer la pensée d'un Aristote « objectif », par opposition à un Aristote « déformé » par ses « interprétations ». Mais plutôt pour substituer à l'aristotélisme une lecture d'Aristote qui radicalise la différence entre la pensée d'Augustin et celle d'Aristote. Lecture qui sera donc bien, elle aussi, une interprétation. Mais qui, loin de ramener le débat sur un unique terrain consensuel (celui de la logique), accusera le litige : celui du logos et de la logique.

Si la pensée augustinienne de la Trinité devait effectivement constituer une révolution en regard non seulement de la logique de l'aristotélisme, mais aussi et plus profondément du logos d'Aristote, notre thèse aurait une seconde conséquence: elle impliquerait que saint Augustin, dans sa critique de l'aristotélisme, dépasse la pensée d'Aristote, qu'il ne critique pourtant pas. Dans cette mesure, ce qui serait en jeu, ce ne serait pas seulement un débat avec la pensée hellénistique restituable dans le cadre d'une histoire 
des idées. Mais un dialogue avec Aristote qu'il nous faudrait reconstruire, et qui engagerait la compréhension d'une révolution philosophique.

La critique la plus conséquente des catégories qu'ait mené Augustin se trouve dans le De Trinitate. C'est sur l'étude de cet ouvrage que nous nous concentrerons donc essentiellement.

Dans un premier temps, nous tenterons toutefois de clarifier, d'un strict point de vue biographique, historique et philologique, la relation que saint Augustin est susceptible d'avoir entretenu avec le texte aristotélicien des Catégories.

Nous tenterons ensuite de déterminer la nature de l'interprétation augustinienne des catégories : nous pourrons alors confirmer une partie de notre hypothèse, à savoir sa teneur hellénistique (en l'occurrence néoplatonicienne et stoïcienne).

Dans un troisième temps, nous confronterons cette interprétation au traité des Catégories d'Aristote : ce qui devrait nous permettre de confirmer l'autre partie de notre hypothèse - à savoir l'irréductibilité du logos aristotélicien à la logique, telle qu'elle fut élaborée dans la pensée hellénistique.

Enfin, nous verrons que si la critique augustinienne des catégories n'atteint pas la pensée d'Aristote, elle n'en conduit pas moins à son dépassement. Nous essaierons alors de montrer que la parole qui tente de nommer la Trinité est peut-être moins la substitution d'une logique à une autre, que l'ouverture d'un nouveau rapport à la parole.

\section{Augustin et le texte des Catégories d'Aristote}

Au livre IV des Confessions, saint Augustin évoque « un certain traité d'Aristote, appelé Les Dix Catégories », qu'il a " eu un jour entre les mains », alors qu'il avait " une vingtaine d'années ». ${ }^{4}$ Augustin ne dit pas qu'il a étudié les Catégories d'Aristote, mais simplement qu'il les a " eues un jour entre les mains » (in manus meas uenissent) - suggérant le caractère circonstanciel et circonstancié de cette lecture. Simple anecdote. Sans compter que le traité d'Aristote semble être lui-même quelque chose d'anecdotique : Augustin ne parle pas des Catégories d'Aristote, mais d' " un certain traité d'Aristote appelé Les Dix Catégories » (Aristotelica quaedam, quas appellant decem categorias) - comme s'il s'agissait d'un ouvrage inconnu et qui aurait besoin d'être identifié. Et, pour le cas où

${ }^{4}$ Saint Augustin, Confessions, IV, XVI, 28, Patrologie Latine, Migne, 1865 (désormais noté PL), t. 32, p. 704 ; trad. fr. P. Cambronne, in Saint Augustin, Euvres, I, Paris, Gallimard (Bibliothèque de la Pléiade), 1998, p. 854. 
l'on n'aurait pas encore compris le caractère pour le moins secondaire de cet ouvrage, Augustin avoue ne s'être jamais vraiment expliqué que les Catégories aient pu présenter de telles difficultés à tous ceux qui les ont étudiées - lui qui, dit-il, les a lues très facilement, et sans se faire aider de professeurs. Et il conclut que cette lecture a non seulement été inutile, mais nuisible - le conduisant à penser Dieu, sinon comme un corps, en tout cas sur le modèle des corps. ${ }^{5}$

Augustin fait également mention des Catégories d'Aristote dans un autre texte : le Contra Iulianum, où se trouve consignée sa polémique avec le pélagien Julien d'Eclane. Dans cet ouvrage, le traité des Catégories est cité à cinq reprises par Augustin : en I, 12 ; II, 37 ; III, 7 ; V, 51 ; et VI, 64. Si la tonalité de l'ouvrage est déjà en soi polémique, le ton sur lequel Augustin se réfère au traité d'Aristote l'est peut-être plus encore. A chaque fois qu'Augustin cite ce traité, c'est pour reprocher à son adversaire de lui vouer une admiration qui confine à l'adoration. Dans ce contexte, les catégories d'Aristote ne semblent être, aux yeux d'Augustin, que des subtilités dans lesquels Julien d'Eclane se perd et, par occasion, cherche à perdre ceux qui voudraient combattre son hérésie. A titre d'exemple, voici ce que dit saint Augustin en VI, 64:

« J'estime qu'avant tout vous vouliez rendre mon langage inintelligible, afin de trouver là une belle occasion d'entasser de profondes ténèbres, à l'aide de toutes les catégories d'Aristote, et de faire applaudir votre génie par ceux qui ne savent même pas ce que vous dites. $»^{6}$

L'usage qu'Augustin fait de ces termes: " catégories d'Aristote », en fait une sorte d'invective qu'il jette à la figure de son adversaire. Cependant, ce qu'Augustin reproche à celui-ci, ce n'est évidemment pas d'avoir lu le traité des Catégories ${ }^{7}$. Ce dont il l'accuse, c'est plutôt de se servir des catégories d'Aristote comme d'une arme visant à réduire au silence son adversaire, en en faisant l'unique critère de tout discours vrai. Cette prétention étant écartée, Augustin ne se refuse pas à engager une discussion sur les catégories elles-mêmes - ce qu'il fait, quoique très brièvement, et sans citer le traité d'Aristote, en V, 21.

${ }^{5}$ Ibid, IV, XVI, 29. PL, p. 704-705 ; trad. fr. p. 854.

${ }^{6}$ Saint Augustin, Contra Iulianum, PL, t. 44, p. 862 ; trad. fr. M. Raulx (dir.), Contre Julien, in Euvres Complètes de saint Augustin, Bar-le-Duc, Louis Guérin, 1871, t. 16, p. 257.

${ }^{7} \mathrm{Du}$ reste, F.-J. Thonnard, dans son article « L'aristotélisme de Julien d'Eclane et saint Augustin » (Revue des études augustiniennes, 1965, vol. IX, n 3-4, p. 296-304), a bien montré que l'interprétation des catégories sur laquelle se fondait le pélagien dans la polémique qui l'opposait à Augustin était loin d'être fidèle à la pensée d'Aristote. 
C'est encore une hérésie qui conduira Augustin à parler une nouvelle fois des catégories, dans le De Trinitate - essentiellement aux livres V et VII. Ce n'est plus contre le pélagisme, mais contre l'arianisme que la critique est menée. Seulement cette fois, Augustin ne peut plus se contenter, comme il le faisait avec Julien d'Eclane, de persifler l'aristotélisme de ses adversaires. C'est un débat de fond avec l'aristotélisme qu'il est ici contraint d'engager. Car si les ariens se réfèrent aux catégories, le concile même qui les a convaincus d'hérésie s'y réfère aussi, au moins implicitement. En effet, pour condamner l'arianisme, qui concevait la différence des personnes de la Trinité comme substantielle, le concile de Nicée, en 325, déclara le Fils «consubstantiel» au Père - en grec: ónoov́otoc. C'est la foi catholique elle-même qui conduit Augustin sur le terrain grec de l'òvoía. ${ }^{8}$

En soi, le terme d'óvoía n'embarrasse pas saint Augustin. Dans le De Trinitate, il écrit en effet, en parlant de Dieu :

« Il n'y a pourtant aucun doute sur le fait qu'il est une "substance" ou,

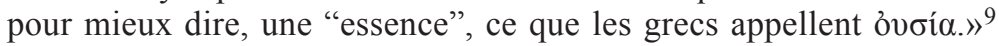

Bien que tous deux puissent traduire le grec ỏvoía, le mot " essence » est préféré au mot «substance ». ${ }^{10}$ Pourquoi ? Dans le mot essentia vibre encore le verbe esse à partir duquel il a été formé, et où saint Augustin entend résonner le sum qui sum par lequel Dieu, dans le texte de l'Exode $(3,14)$, déclare son nom à Moïse. ${ }^{11}$ Essentia peut donc faire directement

${ }^{8}$ C'est bien dans le cadre du Symbole de Nicée que le texte même des livres V à VII du De Trinitate se situe. Le texte est encadré par des citations du Symbole. Au début, en V, VI, 7, Augustin examine l'expression « est égal au Père »; et à la fin, en VII, VI, 12, il cite des passages entiers du Symbole.

${ }^{9}$ De Trinitate, V, II, 3. PL, p. 912 ; trad. fr. S. Dupuy-Trudelle, La Trinité, in Saint Augustin, Euvres, III, Paris, Gallimard (Bibliothèque de la Pléiade), 2002, p. 406.

${ }^{10}$ Sur l'histoire de ces deux traductions du mot òvoía, cf. Jean-François Courtine, «Les traductions latines d' ỏvoía et la compréhension romano-stoïcienne de l'être », in Les catégories de l'être. Etudes de philosophie ancienne et médiévale, Paris, P.U.F. (coll. « Epiméthée »), 2003, p. 11-78.

${ }^{11} \mathrm{Cf}$. notamment De Trinitate, V, II, 3, où le mot essentia est rapporté au verbe esse, lui-même directement mis en relation avec le Sum qui sum de l'Exode 3, $14:$ «... on a tiré le mot essentia ("essence") de esse ("être"). Et qui "est" plus que celui qui a dit à son serviteur : "Je suis celui qui suis" " (... ab eo quod est esse dicta est essentia. Et quis magis est, quam ille qui dixit famulo suo Moysi : "Ego sum qui sum ») (PL, p. 912 ; trad. fr. p. 406). Cf. aussi et surtout VII, V, 10, où Augustin, se fondant là encore sur le texte de l'Exode, va jusqu'à envisager de réserver le mot essentia à Dieu : « ... de sorte, peut-être, que Dieu seul mérite le terme d' "essence". C'est que seul il est véritablement, parce qu'il est immuable, et qu'il s'est appelé ainsi devant son serviteur Moïse, quand il 
signe vers les Ecritures. En revanche, substantia appelle quasi inévitablement son corollaire : accidens. Or il n'y a pas d'accidents en Dieu : Dieu est un et immuable, alors que l'accident suppose la multiplicité et le changement. Substantia sous-entend ainsi une logique du multiple qui est étrangère à Dieu. On pourra toujours dire de Dieu qu'il est une substance sans accident ; mais justement, il faudra nécessairement apporter cette précision, dont le terme essentia, lui, peut parfaitement se passer. ${ }^{12}$

Les choses auraient pu en rester là : préférer le mot essentia au mot substantia, et s'en tenir à l'unité dans laquelle le premier met de plein pied, loin de la logique du multiple que sous-entend le second.

Cependant, c'est précisément sur le terrain de la logique du multiple que 1'ó $\mu$ oov́otos du concile de Nicée semble nous ramener. En effet,

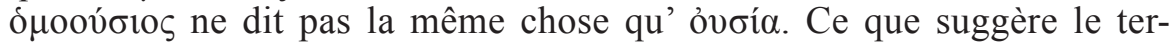
me, ce n'est pas la pure et simple unité : dans ce cas, on n'aurait qu'à

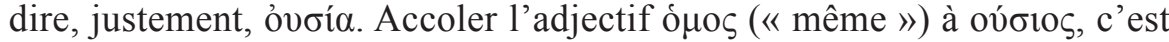
présupposer une multiplicité que l'adjectif ómo $\varsigma$ vise précisément à rassembler. Comment penser cette multiplicité sur un autre modèle que celui de la substance et de ses accidents ? Pour y répondre, il faut s'engager dans une critique de ce modèle. Autrement dit, il faut critiquer la logique catégoriale.

C'est ainsi qu'Augustin, dans le De Trinitate, procède à un examen rigoureux et détaillé des catégories.

Cependant, les catégories qu'il examine sont-elles bien celles d'Aristote ? La logique catégoriale, si elle est d'origine aristotélicienne, dépasse largement le cadre des seuls traités d'Aristote. Commentée, reprise, transformée, elle est un bien et presque un lieu commun de la philosophie antique tardive. ${ }^{13}$ Dans les deux livres du De Trinitate qu'il consacre à la critique du catégorial, Augustin n'éprouve pas le besoin de citer, ne serait-ce qu'une seule fois, le nom du Stagirite.

On pourrait évidemment se demander si Augustin a bien lu les Catégories d'Aristote, ainsi qu'il l'affirme (non sans la prise de distance qu'on a vue) dans les Confessions. On considère habituellement que l'ouvrage qu'Augustin a eu entre les mains est la traduction latine des Catégories

a dit : "Je suis celui qui suis" " (... ita ut fortasse solum Deum dici oporteat essentiam. Est enim vere solus, quia incommutabilis est, idque nomen suum famulo suo Moysi enuntiavit, cum ait : "Ego sum qui sum ») (PL, p. 942 ; trad. fr. p. 458). Pour une analyse intégrale et détaillée des passages où Augustin cite ce passage de l'Exode 3, 14, cf. Emilie Zum Brunn, "L'exégèse augustinienne de "Ego sum qui sum" et la "métaphysique de l'Exode" ", in Dieu et l'être, Exégèse d'Exode 3, 14 et de Coran, 20, 11-24, Paris, Institut d'Etudes Augustiniennes, 1978, p. 141-164.

12 De Trinitate, VII, V, 10. PL, p. 942-943 ; trad. fr. p. 457-458.

13 Cf. C. Stead, Divine Substance, Oxford, Clarendon Press, 1977, p. 110-118. 
d'Aristote par Victorinus ${ }^{14}$. L'argument souvent invoqué, à la suite de F. Bömer, pour étayer cette hypothèse est l'ordre d'énumération des catégories : dans le De Trinitate, saint Augustin modifie légèrement l'ordre d'énumération aristotélicien, et fait passer le temps et le lieu après la position et la possession ${ }^{15}$; or la même interversion aurait été opérée par Victorinus dans sa traduction des Catégories, qui a au demeurant été perdue. ${ }^{16}$ Cependant, comme le note P. Hadot, ${ }^{17}$ l'énumération des catégories que propose Victorinus dans son commentaire du De inventione de Cicéron ne comporte pas cette interversion. Mais plus essentiellement, il semble avoir échappé aux commentateurs que l'énumération augustinienne des catégories dans les Confessions ne maintient pas cette interversion : ici la position et la possession viennent bien, comme dans l'énumération aristotélicienne, après le lieu et le temps. ${ }^{18}$ Et du reste, certains commentateurs, comme L. Minio-Paluello ou P. Hadot ${ }^{19}$, contestent qu'Augustin ait pu lire la traduction de Victorinus " sans raconter dans ses Confessions qu'elle venait du rhéteur converti $»^{20}$. Ce qui peut signifier qu'Augustin n'a pas lu les Catégories d'Aristote dans la traduction de Victorinus. Mais ce qui peut laisser entendre aussi qu'Augustin ne les a tout simplement pas lues ${ }^{21}$.

Il faut bien avouer que les traces qui pourraient témoigner d'une authentique lecture du texte d'Aristote sont bien faibles dans l'œuvre de saint Augustin. Mais indépendamment de la question de savoir si cette lecture est de première ou de seconde main, un constat s'impose : la lecture au-

14 Cf. P. Courcelle, Les lettres grecques en occident. De Macrobe à Cassiodore. Paris, 1948, p. 156, et H.-I. Marou, Saint Augustin et la fin de la culture antique. Paris, 1948 , p. 34.

${ }^{15}$ Cf. par exemple De Trinitate, V, VII, 8.

${ }^{16}$ F. Bömer, Der lateinische Neuplatonismus und Neupythagoreismus und Claudianus Mamertus in Sprache und Philosophie, Leibzig, 1936, p. 87-88 - cité par P. Hadot, Marius Victorinus, Paris, Etudes Augustiniennes, 1971, p. 188.

${ }^{17}$ P. Hadot, op. cit., p. 188.

18 Cf. Confessions, IV, XVI, 28 (PL, p. 704 ; trad. fr. p. 854) où, cette fois, Augustin fait passer la qualité avant la quantité, mais situe bien le lieu et le temps avant la position et la possession : "Cet ouvrage, me semblait-il, parlait assez clairement de la notion de "substance" - l'homme, par exemple -, et d' "accidents" - ainsi pour l'homme, sa configuration, sa taille (combien de pieds mesure-t-il ?), sa parenté (de qui est-il le frère ?), son lieu de séjour, sa date de naissance, sa position (est-il assis ou couché ?), son état (est-il chaussé ou armé ?), son action, sa "passion" - et tout ce qui, dans les neuf genres cités (j'en ai donné des exemples) et celui-là même de substance, peut être trouvé à l'infini. »

${ }^{19}$ L. Minio-Paluello, The Text of the Categoriae : the latin tradition, in Classical Quarterly, t. XXXIX, 1945, p. 63-74, et P. Hadot, op. cit., p. 188.

20 P. Hadot, op. cit., p. 188.

${ }^{21}$ L. Minio-Paluello pense qu'Augustin a pu lire les Categoriae decem, qu'il attribue à Albinus. 
gustinienne des catégories est, comme on va le voir, très profondément marquée par le néoplatonisme et le stoïcisme.

\section{L'interprétation augustinienne des catégories}

Sa critique des catégories, Augustin ne lui accorde, au livre V du De Trinitate, qu'une très brève introduction. Celle-ci consiste à délimiter le domaine d'application des catégories. Augustin distingue d'abord trois ordres : le corporel, l'intellectuel (qui est, précise-t-il, « ce qu'il y a de meilleur en nous »), et Dieu. Et il conclut :

« Donc, ce que nous ne trouvons pas dans ce qu'il y a de meilleur en nous, nous ne devons pas le chercher dans celui qui est, de loin, meilleur que ce qu'il y a de meilleur en nous. $»^{22}$

Autrement dit, nous ne devons pas appliquer à Dieu des déterminations corporelles que nous n'appliquons pas même à notre intellect. De quelles déterminations s'agit-il ? Des catégories. L'inadéquation des catégories pour penser l'intellect sera établie au livre X. Ici, saint Augustin en nie directement la pertinence pour penser Dieu. Cependant, cette négation garde la trace de ce qu'elle nie :

«Aussi devons-nous saisir intellectuellement Dieu comme bon sans qualité, grand sans quantité, créateur sans indigence, présent sans localisation, contenant tout sans possession, tout entier partout sans lieu, éternel sans temps, faisant les choses muables sans mutabilité en lui, et ne subissant rien. $»^{23}$

Abstraction faite de la substance, qui sera examinée à part, la liste des catégories est complète : qualité, quantité, relation (signifiée par le terme d'indigence), position (appelée ici localisation), possession, lieu, temps, action et passion. ${ }^{24}$ Tous ces chefs de prédication sont niés de Dieu. Niés, car ils appartiennent exclusivement, selon Augustin, au domaine corporel. Ce présupposé s'éclairera par la suite. En tout cas, c'est au travers de cette négation même que quelque chose de Dieu se fait jour. Augustin ne

22 Saint Augustin, De Trinitate, V, I, 2. PL, p. 912 ; trad. fr. p. 405.

${ }^{23}$ Idem. PL, p. 912 ; trad. fr. p. 405-406.

${ }^{24} \mathrm{Cf}$. Aristote, Catégories, IV, $1 \mathrm{~b} 25$ sq., qui énonce la liste des dix catégories : óvoía,

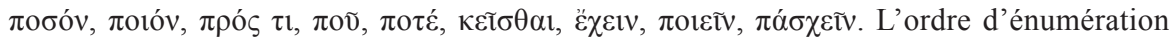
est ici doublement modifié par Augustin : il fait passer non seulement la position et la possession avant le temps et le lieu, mais également la qualité avant la quantité. Cette dernière interversion sera corrigée en V, VII, 8 et, comme on l'a vu, la première le sera dans les Confessions, IV, XVI, 28. 
dit pas en effet que nous devons penser Dieu comme sans qualité, sans quantité, etc. - mais que nous devons le penser comme bon sans qualité, grand sans quantité. Tout se passe comme si Dieu était d'essence catégoriale. Mais le comme si fait toute la différence.

Ce comme si trouve son explication un peu plus loin. Augustin commence par distinguer la chose sensible de la notion sous laquelle elle est comprise (par exemple la notion de grandeur) :

« La véritable grandeur est celle par laquelle non seulement une grande maison est grande, une grande montagne est grande, mais aussi est grand tout ce qui est dit grand, de sorte qu'autre chose est la grandeur, autre chose est ce qu'elle rend grand. $»^{25}$

Augustin comprend les catégories comme des notions. Jusque là, Augustin et Aristote pourraient tomber d'accord. ${ }^{26}$ Cependant, là où ils diffèrent radicalement, c'est dans la détermination du rapport entre la chose et sa catégorie. Augustin détermine en effet ce rapport en recourant à une notion pour le moins non-aristotélicienne : la participation. Quelques lignes avant le texte précité, Augustin note en effet :

« Car, dans les réalités qui sont grandes par participation à la grandeur (participatione magnitudinis), autre chose est d'être, autre chose est d'être grand. $»^{27}$

Une chose participe à sa catégorie. Cette interprétation si peu aristotélicienne d'Aristote est d'origine néoplatonicienne. L'illustration la plus claire s'en trouve sans doute dans l'Isagoge de Porphyre. En II, 6, celui-ci détermine, au travers de son célèbre " arbre », le rapport hiérarchique des espèces et des genres. Au sommet de l'arbre, on trouve la catégorie, entendue comme genre suprême. Il reste alors à déterminer le rapport que l'individu entretient avec l'arbre tout entier - et donc, à terme, avec la catégorie. C'est ce qui est fait en II, 12, où Porphyre écrit :

« C'est par la participation à l'espèce que les hommes multiples constituent l'homme un, tandis que par les individus cet homme unique et commun devient plusieurs. $»^{28}$

${ }^{25}$ De Trinitate, V, X, 11. PL, p. 918 ; trad. fr. p. 415.

26 Pour Aristote, les catégories sont des genres : on en trouve la confirmation, par exemple, en Métaphysique, I, 3 (1054b35), où Aristote explique que deux choses comprises sous la même catégorie sont identiques par la genre - catégorie et genre étant par conséquent synonymes.

${ }^{27}$ De Trinitate, V, X, 11. PL, p. 918 ; trad. fr. p. 415.

28 Porphyre, Isagoge, II, 12, trad. fr. A. de Libera, Paris, Vrin, 1998, p. 8. 
Ce qui est dit ici de l'espèce vaut aussi bien pour la catégorie, qui ne s'en distingue que par sa plus grande généralité. Or le mode sur lequel l'individu s'y rapporte est la participation. Le terme grec est $\mu \varepsilon \tau o v \sigma i ́ \alpha$, que le latin traduira par participatione. On pourrait évidemment discuter la fidélité de cette traduction. Quoi qu'il en soit, c'est sous le nom de participation que la $\mu \varepsilon \tau o v \sigma i ́ \alpha$ porphyrienne, c'est-à-dire le rapport de l'individu à sa notion, se diffusera dans le monde romain. Et c'est sous ce nom qu' on la trouve déjà sous la plume d'Augustin.

Si la prédication catégoriale implique la participation, on comprend rétrospectivement pourquoi saint Augustin pouvait dire de Dieu qu'il est grand sans quantité. Grand sans quantité signifie : grand sans participation à la grandeur. Dieu ne participe pas à la grandeur, il est grandeur. Non pas que Dieu s'identifie aux catégories elles-mêmes : il est encore au-delà. Saint Augustin note en effet :

«Dieu donc est grand par la grandeur qui fait qu'il est lui-même sa propre grandeur. $»^{29}$

Dieu ne participe pas à la grandeur. Mais il n'est pas non plus la grandeur elle-même. La grandeur à laquelle Dieu s'identifie est d'une toute autre nature que la notion de grandeur qui s'applique aux choses. On pourrait dire que Dieu a sa grandeur bien à lui. Ou plutôt qu'il est sa grandeur bien à lui. ${ }^{30}$

Dès lors, qu'est-ce qui nous autorise encore à parler de Dieu en termes de grandeur - comme s'il s'agissait, peu ou prou, de la même grandeur que celle à laquelle participent les choses ? L'impossibilité d'en parler autrement. Dieu est dit grand, précise Augustin, « au sens figuré et par ressemblance » (translate ac per similitudines) ${ }^{31}$. Quand donc Augustin dit de Dieu qu'il est grand sans quantité, ce n'est pas seulement la quantité qu'il faudrait nier - c'est-à-dire la participation ; il faudrait nier aussi la grandeur - c'est-à-dire la catégorie elle-même. Mais avec cette seconde négation, on ne pourrait plus rien dire. Tout se passe donc comme si Dieu était identique à ses catégories. Comme si : c'est-à-dire qu'il ne l'est que par ressemblance. En réalité, Dieu est supracatégorial.

${ }^{29}$ De Trinitate, V, X, 11. PL, P. 918 ; trad. fr. p. 416. Cf. également Confessions, IV, xvi, 29 où, après avoir évoqué sa lecture des Catégories, Augustin écrit, en s'adressant à Dieu : «tu es toi-même ta propre grandeur et ta propre beauté » (PL, p. 705 ; trad. fr. p. 854).

${ }^{30}$ Cette thèse de l'identité de Dieu et de ses attributs, mainte fois affirmée par saint Augustin (notamment, pour le texte qui nous occupe, en VII, V, 10), trouve sans doute sa formulation la plus synthétique dans la Cité de Dieu, XI, X, 1 : «Dieu est ce qu'il a ».

31 De Trinitate, V, VIII, 9. PL, p. 917 ; trad. fr. p. 414. 
Ce qui a été ainsi soumis à une critique, ce sont les neuf catégories qui peuvent être attribuées à un sujet. Reste évidemment à déterminer le sens qu'il convient d'accorder au sujet lui-même, c'est-à-dire à la substance. Cette critique est menée dans la seconde partie du livre VII. Et c'est là que la lecture néoplatonicienne des catégories se double d'une lecture stoïcienne.

Voici ce qu'écrit saint Augustin au sujet de la substance:

« Ce mot (substance) désigne habituellement à juste titre ces réalités, ces sujets dans lesquels sont les choses qui sont attribuées à un sujet, comme la couleur ou la forme dans le corps. Car le corps subsiste, et c'est pourquoi il est substance. $»^{32}$

Conformément à sa racine, qu'Augustin a expressément dégagée au $\S 9$, le mot substantia est entendu à partir d'un subsistere. Autrement dit, la substance est comprise comme sujet et comme substrat. Que faut-il entendre par là ? En guise de précision, Augustin n'apporte qu'un exemple : celui des corps. Cet exemple serait-il alors un archétype ? Tel semble bien être le cas. En V, I, 2, lorsque saint Augustin dit de Dieu qu'il est «bon sans qualité, grand sans quantité... », la négation des catégories n'est justifiée que par leur champ d'application : le domaine corporel. Si bien que les catégories, et au premier rang d'entre elles la substance, semblent être constitutivement liées à la corporéité. C'est pourquoi elles ne sauraient pas davantage s'appliquer à l'intellect : en $\mathrm{X}, \mathrm{X}, 15$, lorsque saint Augustin examine les interprétations matérialistes de la pensée, c'est encore à la logique catégoriale qu'il impute l'erreur matérialiste. Si une telle logique conduit nécessairement à considérer l'esprit comme une simple qualité corporelle, c'est qu'elle implique, dit saint Augustin, de considérer l'esprit « comme étant dans un sujet». Mais pourquoi ? Parce qu'aussi longtemps que sujet signifiera corps, l'esprit ne pourra pas être un sujet - mais tout au plus la qualité d'un sujet, c'est-à-dire la qualité d'un corps. La synonymie du sujet (autrement dit de la substance) et de la corporéité ne semble pas même faire question.

Cette interprétation de la substance à partir de la notion de substrat corporel se rencontre chez les stoïciens. Dans son traité Sur les catégories d'Aristote (48, 11-16), Simplicius rappelle que pour les stoïciens, la substance comprise comme substrat est double : d'une part, matière inqualifiée, et d'autre part, matière disposée à recevoir des qualités. ${ }^{33}$ Ici,

32 Ibid., VII, V, 10. PL, p. 942 ; trad. fr. p. 457-458.

${ }^{33}$ Pour une étude de l'interprétation stoïcienne de la substance à partir de la notion de corporéité, cf. E. Weil, « Le matérialisme des stoïciens », in Essais et conférences, 
Simplicius rapproche la doctrine stoïcienne de celle d'Aristote. La doctrine stoïcienne peut apparemment se réclamer de la pensée aristotélicienne de

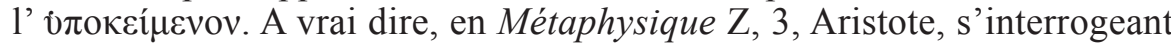

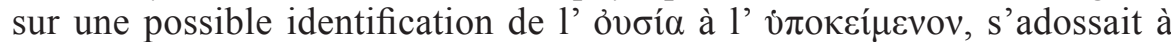
la distinction implicite de deux plans : le catégorial, et l'hylémorphique.

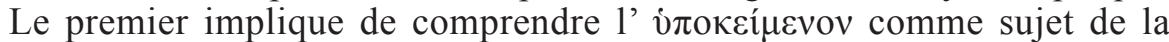
prédication ; le second, de le comprendre comme matière. ${ }^{34}$ Le stoïcisme procède à une fusion de ces deux plans : le sujet de la prédication est lui-même compris comme matière. Et comme on ne saurait assigner à la prédication un sujet qui serait lui-même purement indéterminé, puisque c'est bien de lui qu'on affirme telle ou telle détermination, le sujet doit être pensé comme matière qualifiée, c'est-à-dire comme corps. Le stoïcisme

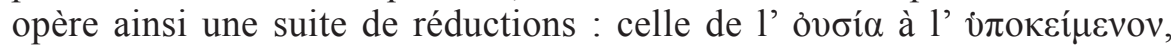

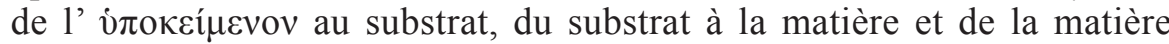
au corps.

Cette interprétation stoïcienne de la substance comme substrat corporel explique donc rétroactivement qu'Augustin ait pu, en V, I, 2, limiter le catégorial au domaine corporel. C'est ainsi que le catégorial était nommé par Augustin " l'aune habituelle des réalités visibles, muables ou mortelles ». " Habituelle » signifie donc : corporelle. Et « l'aune », ce sont les catégories.

Résumons-nous. Les catégories qui sont prédiquées de la substance sont, pour Augustin, des notions auxquelles la substance participe. La substance, quant à elle, est pensée comme corporéité du corps. L'interprétation augustinienne du catégorial se fait sur la base d'un néoplatonisme doublé de stoïcisme. C'est précisément ce catégorial-là qu'Augustin critique et dépasse dans sa réflexion sur la Trinité. Jusqu'à quel point le catégorial s'y réduit-il ?

Paris, Vrin, 1991, p. 106-123 (cf. en particulier p. 113 : « Il faudra donc parler, comme nous venons de le proposer, de corporalisme, non de matérialisme. Tout ce qui est corps, est ; tout ce qui est, est corps : voilà la thèse de l'Ecole »).

${ }^{34}$ Sur ce point à la fois complexe et décisif, cf. R. Boehm, La Métaphysique d'Aristote. Le Fondamental et l'Essential, Paris, Gallimard, 1976, dont on trouvera une synthèse très complète et très dense dans l'article du même auteur, « Le fondamental est-il l'essentiel ? (Aristote, Métaphysique Z 3) ", in Revue Philosophique de Louvain, Troisième série, Tome 64, n 83, 1966, p. 373-389. Les acquis des recherches de R. Boehm ont été repris et reconduits à la distinction du catégorial et de l'hylémorphique, et par suite du $\lambda$ ó ${ }^{\circ} \varsigma$

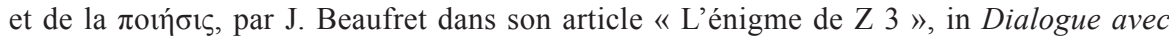
Heidegger, t. 4, Le chemin de Heidegger, Paris, Les Editions de Minuit, 1985, p. 15-31. 


\section{L'interprétation augustinienne à l'épreuve du logos apophantikos}

Le catégorial est, dans la pensée d'Aristote, l'un des sens de ce que le Stagirite appelle l'ốv ᄁ̣̂ ơv. Ce qui signifie littéralement : l'étant en tant qu'étant. Autrement dit : l'angle sous lequel l'étant est à proprement parler. C'est ce que confirme par exemple la Métaphysique où, en $\Delta, 7$, Aristote explique que le catégorial est, avec l'accident, la vérité, et la puissance et l'acte, l'un des quatre sens fondamentaux de l'être.

Le traité des Catégories pense prioritairement l'étant à partir de la notion d' óvoía. Que faut-il entendre par là ? Voici ce qu'en dit Aristote en $2 \mathrm{a} 11$ :

« L'ỏvøía au sens souverain, premier et principal, ce n'est ni ce qui se dit selon un sujet, ni ce qui est dans un sujet : c'est par exemple cet homme-ci, ce cheval-ci. »

Ce que la tradition retiendra de ce passage, c'est que l' óvoía signifie prioritairement l'individu. Il est indéniable que cet homme-ci ou ce cheval-ci sont individuels. Mais la notion d'individu est-elle vraiment à même de rendre fidèlement compte de ce que disent les expressions :

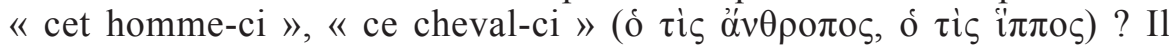
arrive effectivement à Aristote d'en parler en termes d'unité numérique $(\ddot{\varepsilon} \nu \alpha \hat{\rho} \mathrm{i} \theta \mu \tilde{\omega})$. Mais cette terminologie est toujours contextuelle : Aristote l'emploie par exemple lorsqu'il veut dire que l' ỏvбía demeure la même en dépit du changement (4b17). En-dehors de ces occurrences contextuelles, l'appellation qu'il privilégie est : $\tau o ́ \delta \varepsilon \tau \imath$ que l'on pourrait traduire par : « ceci que voici ». Dès lors, la compréhension que l'on a de l' óvбía, du moins dans son sens premier et principal, dépend de l'entente que l'on a de ce déictique.

L'interprétation qu'en propose indirectement saint Augustin, au travers du stoïcisme, consiste à identifier ce qu'indique le déictique au corps. Evidemment, on ne saurait montrer du doigt une idée ou une émotion. Mais peut-on affirmer pour autant que ce qu'on montre, quand on le montre, doit avant tout être pensé comme corporéité d'un corps ? Aristote emploie le mot « corps » $(\sigma \tilde{\omega} \mu \alpha)$ dans un contexte unique : lorsqu'il dit par exemple

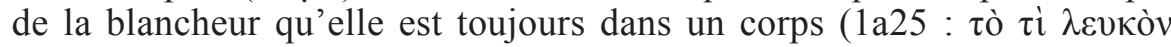

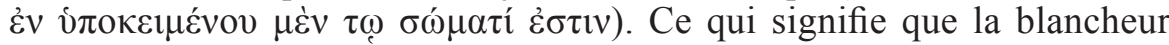

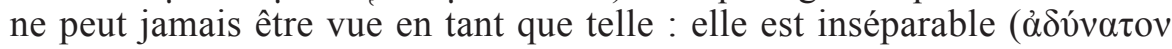
$\chi \omega \rho i ́ s)$ de ce qui est blanc. Or ce qui est blanc, c'est toujours quelque chose de corporel. Mais le niveau de discours auquel on se situe quand on parle de blancheur est le niveau le plus rudimentaire : c'est celui qui consiste à énumérer ce qui est dans une chose - c'est-à-dire ce qu'une chose est de manière purement accidentelle. En outre, ce qu'Aristote dit 
de la blancheur, il ne le dit pas de l'accident en général : par exemple, il est accidentel que tel homme soit grammairien, mais la grammaire n'est bien entendu pas dans le corps de cet homme. La corporéité n'est donc $q u$ 'un des éléments auxquels certains accidents se rapportent. Ce qui signifie à peu près ceci : le déictique, lorsqu'il est envisagé sous son jour le plus accidentel, doit parfois être envisagé comme corps.

Mais c'est sous un tout autre jour que le déictique peut pleinement se déployer. Le déictique est le premier sens de l'ỏvoía. Mais ce premier sens est inséparable d'un second, qu'Aristote définit ainsi :

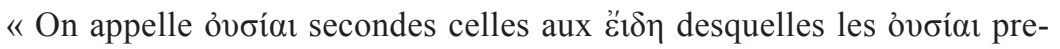
mières appartiennent. »

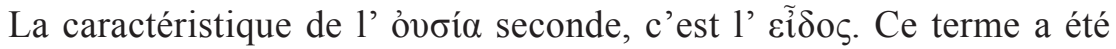
traduit en latin par species, qui a donné notre mot espèce. Ce qu'ont en commun le terme grec et sa traduction latine, c'est d'appartenir au lexique de la vision. L' óvoía seconde est ainsi la dimension au sein de laquelle la chose est vue comme ce qu'elle est. Cette dimension est à ce point inséparable de la chose, qu'elle lui donne jusqu'à son nom. C'est ce que note Aristote en $2 \mathrm{a} 21$ :

«Homme est dit du sujet, à savoir cet homme-ci, et on lui en attribue le nom. »

Autrement dit, pour désigner untel ou untel, on dira : « cet homme-ci ». L'humanité percera jusque dans le déictique. Nous avons appelé cela la dimension de visibilité de ce qu'indique le déictique. On pourrait parler aussi en termes d'horizon. Le mot qu'emploie Aristote pour en parler est l'adjectif $\delta \eta \lambda$ ó $\varsigma$, que l'on pourrait rendre en français par l'adjectif " manifeste ». Ce qui nous conduirait à traduire ainsi le passage suivant, qui se situe en 2 b32 :

« Parmi tous les catégorèmes, les voíal secondes sont les seuls à rendre manifestes les voíaı premières. »

Ainsi, l' cĩ $\delta o s$, avant que d'être une espèce qui s'articule logiquement à un genre, est ce qui rend manifeste la chose dans ce qu'elle est - par exemple cet homme-ci dans son humanité. Bien entendu, la chose ne se

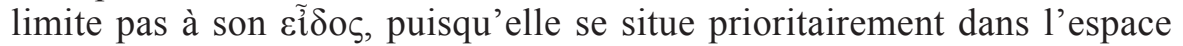
ouvert par le déictique. Cependant, l' $\varepsilon 1 \delta o s$, autrement dit l'ỏvoía seconde, en est l'horizon de manifestation.

Nous avons vu que l' ỏvoía n'est pas un corps ; qu'elle est bien plutôt, prioritairement, la présence d'une chose sur un mode déictique, qui se

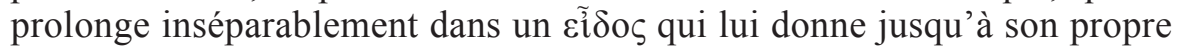


nom. L'interprétation stoïcienne de la substance comme corps consiste donc à greffer un concept physique sur une interprétation logique et formelle du discours.

Cependant, ce qui conduisait Augustin à penser la substance comme corporéité du corps, c'était, plus profondément que l'idée de corps, l'idée de subsistance. Or à la fin du chapitre des Catégories consacré à l' ovoía, Aristote semble autoriser cette interprétation de l' ovoía comme support du changement. En 4a11, il caractérise en effet l' óvoía comme " apte à

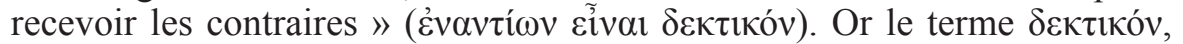
que nous traduisons par " apte à recevoir ", pourrait suggérer l'idée de vase ou de réceptacle. Aristote lui-même, dans la Politique (en 1290b28), emploie ce terme en parlant du ventre, comme " apte à recevoir la nourriture ». L' óvoí $\alpha$ recevrait-elle le changement comme le ventre reçoit la nourriture ? Est-elle le substrat identique et impassible du changement ? Aristote écarte expressément cette idée : le propre de l'ỏvoía n'est pas de rester impassible, mais au contraire d'être capable de changer. Car pouvoir changer, c'est pouvoir se maintenir au cœur même du changement. Etre apte à recevoir les contraires ne signifie donc pas, pour l' ỏvoía, leur demeurer extérieure, mais être capable de les assumer en soi-même - donc, si l'on peut dire, d'être alternativement ces contraires sans pour autant cesser d'être soi-même. Ce qu'Aristote, en $4 \mathrm{~b} 13$, résume en une formule synthétique :

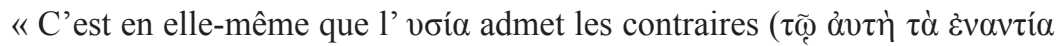
$\delta \varepsilon \dot{\chi} \chi \varepsilon \sigma \theta \alpha)$. »

Reste à déterminer une dernière chose : le rapport que l'v vía entretient aux autres catégories. Ce rapport, Augustin le pensait de manière néoplatonicienne, à partir de la notion de participation. Inutile de préciser que cette notion est, au moins dans ce contexte, on ne peut plus étrangère à la pensée d'Aristote. Mais alors, comment Aristote détermine-t-il ce rapport ? Le traité des Catégories ne le dit pas. Et ce, pour une raison qui lui est constitutive : les catégories, dit Aristote, sont des « expressions

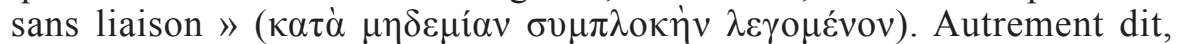
le temps n'est pas encore le temporel, le lieu n'est pas encore le local, etc. Le catégorial comme liaison ne sera abordé que dans le traité qui fait immédiatement suite aux Catégories : le ПEPI EPMHNEIA, De l'interprétation. Au chapitre I, Aristote explique que la liaison se fait par $\sigma v ́ v \theta \varepsilon \sigma ı \varsigma$ et $\delta 1 \alpha i ́ \rho \varepsilon \sigma ı \varsigma$ - disons : par rassemblement et séparation. Que faut-il entendre par là ? Ces termes, Aristote les rapporte directement, dès le chapitre I, à la question du vrai et du faux. D'ordinaire, on les comprend donc à partir de la proposition - le rassemblement étant reconduit à l'affirmation, la séparation à la négation. Réfuter cette interprétation courante nous entraînerait trop loin - c'est-à-dire au moins dans une 
confrontation de Métaphysique, E4 et $\Theta 10$. Pour nous en tenir au traité De l'interprétation, Aristote répond à la question du sens de la liaison par

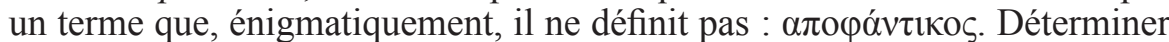
sérieusement le sens de ce terme nous entraînerait sans doute encore plus loin, et impliquerait au fond un parcours critique de l'ensemble de l'histoire de la vérité dans la tradition métaphysique. Tenons-nous en à une remarque lexicale. Le premier sens que le dictionnaire Bailly accorde au verbe

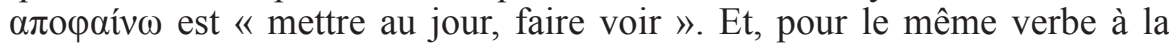
voie moyenne : " se montrer ». Evidemment, rien ne garantit que le sens qu'un dictionnaire donne comme premier soit le bon. Ce sens fait cependant étrangement écho à ce qu'Aristote disait de l' voía seconde - à savoir qu'elle rendait « manifeste » $(\delta \tilde{\eta} \lambda \circ \varsigma)$ l' ỏvoí $\alpha$ première. Dès lors, le rapport de l' óvoía et du catégorial n'est-il pas un rapport de manifestation et de monstration ? Très concrètement, cela signifierait que, lorsque je dis par exemple : «l'arbre est en fleur», je n'accole pas simplement le prédicat « en fleurs » au sujet « arbre », mais que je montre l'arbre sous un certain jour, celui de son être-en-fleur (donc, d'un point de vue catégorial, sous le jour de son $\pi$ otóv, de son « comment ? »), plutôt que, par exemple, sous celui son être-haut-de-trois-mètres (donc de son $\pi$ ó oov, de son « combien ? »). L'apophantique consisterait alors à montrer, par la parole, l'horizon au sein duquel la chose devient visible à chaque fois sous un certain jour.

Evidemment, une telle interprétation de l'apophantique pourrait difficilement être confirmée par des déclarations expresses d'Aristote. Elle n'est qu'une interprétation. Et à vrai dire, l'ensemble de ce qui vient d'être dit d'Aristote procède de présupposés qui, en soi, peuvent tout à fait être contestés. Mais ces présupposés ont peut-être le mérite de radicaliser la différence du $\lambda$ ó ${ }_{0} \varsigma$ aristotélicien et de la parole augustinienne. Le but n'étant pas de montrer qu'Augustin n'aurait pas bien compris Aristote : mais plutôt, ainsi qu'on l'a vu en introduction, de faire d'autant mieux ressortir la singularité de la réflexion augustinienne sur la Trinité. Si cette pensée ne critique pas à proprement parler les catégories d'Aristote, mais plutôt leur entente affaiblie, elle n'en demeure pas moins révolutionnaire par rapport à l'apophantique. C'est ce que nous allons essayer de montrer à présent.

\section{Ousia et relation : d'une propriété apophatique de la parole}

Dès le début du De trinitate, l'apophantique se trouve implicitement - mais non moins décisivement - écarté. En I, I, 3, saint Augustin définit ainsi le projet même de son ouvrage :

«Aussi est-il nécessaire de purifier notre esprit pour qu'il puisse voir de manière ineffable l'ineffable. $»^{35}$

${ }^{35}$ De Trinitate, I, I, 3. PL, p. 821 ; trad. fr. p. 253. 
Il est d'abord remarquable que l'énoncé du projet du De Trinitate mette en jeu le rapport de la parole et de la vision, si essentiel à l'apophantique. Cependant, cette vision ne se déploie pas, comme chez Aristote, à partir d'une parole qui y ouvrirait. Au contraire, ce qui est vu ne peut être vu que dans un dépassement de la parole. Autrement dit, la parole continue bien de déterminer la vision, mais de manière négative comme ineffabilité de la vision.

Cette ineffabilité peut se traduire, comme on l'a vu, par la négation des catégories : Dieu doit être dit bon sans qualité, grand sans quantité, etc. Nier ainsi les catégories, c'est nier les conditions ordinaires du discours. On pourrait alors conclure un peu hâtivement à la simple substitution, chez Augustin, de l'apophatique à l'apophantique, c'est-à-dire de la négation à la monstration. Mais l'approche de l'ineffable ne s'arrête pas là. La négation des catégories se double du renouvellement de l'une d'entre elles : la catégorie de la relation. Ce renouvellement de la catégorie de la relation vient-il compléter positivement la critique simplement négative des catégories ? Une approche positive viendrait-elle prendre le relais d'une approche d'abord simplement négative ? L'ineffable pourrait-il, contre toute attente, être dit ?

Le renouvellement de la catégorie de la relation se fait dans le cadre d'un débat avec l'arianisme. Les Ariens prétendent en effet que tout ce qui se dit de Dieu se dit selon la substance. Car selon eux, tout prédicat est soit substantiel, soit accidentel. Mais comme il n'y a pas d'accident en Dieu, ce qui se dit de lui se trouve limité à la première branche de l'alternative.

Attribuer un statut substantiel à tout prédicat divin vise à terme, pour les Ariens, à établir que la différence du Père et du Fils est elle-même une différence substantielle. Car les prédicats qui s'appliquent au Père et au Fils ne sont pas les mêmes : ainsi, le Père sera dit "inengendré ", alors que le Fils, lui, sera dit «engendré ». Si deux prédicats différents sont attribués substantiellement au Père et au Fils, c'est que le Père et le Fils sont eux-mêmes deux substances différentes.

La réponse d'Augustin va consister à substituer à l'alternative « substance - accident » une autre alternative : l'alternative « substance - relation ». En V, V, 6, Augustin écrit :

« Mais en Dieu rien n'est dit selon l'accident parce que rien en lui n'est muable ; sans que cependant tout ce qui est dit de lui le soit selon la substance. Car certaines choses sont dites relativement à quelque chose, comme le Père relativement au Fils et le Fils relativement au Père. $»^{36}$

${ }^{36}$ De Trinitate, V, V, 6. PL, p. 914 ; trad. fr. p. 409. 
« ... relativement à quelque chose » : en latin ad aliquid, qui est la traduction littérale du grec $\pi \rho{ } \varsigma \tau$. Mais cette nouvelle alternative en est-elle vraiment une ? Le $\pi \rho o ́ \varsigma \tau \imath$, le relatif, n'est-il pas du côté de l'accident ? La réponse est : oui pour les choses créées ; mais non pour Dieu. Dans les choses créées, la relation est accidentelle : par exemple, "père » est un prédicat relatif, car il est accidentel pour un homme d'être père. En Dieu, au contraire, la relation est éternelle : c'est de toute éternité que le Fils est engendré.

Ainsi se fait jour une nouvelle pensée de la relation : la relation trinitaire n'est ni substantielle, ni accidentelle. Mais à quoi tient précisément cette nouveauté ?

Ce qui n'est ni substantiel ni accidentel transgresse l'alternative dans laquelle se tient la logique attributive - y compris chez Aristote. Rappelons le passage du traité des Catégories déjà cité (2a11) :

«L'ỏvoía au sens souverain, premier et principal, ce n'est ni ce qui se dit selon un sujet, ni ce qui est dans un sujet : c'est par exemple cet homme-ci, ce cheval-ci. »

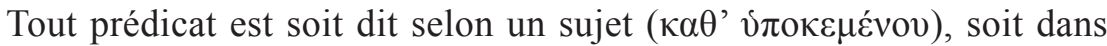

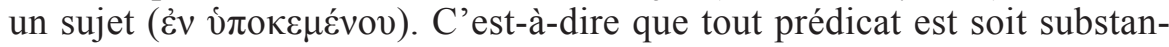
tiel, soit accidentel. En transgressant cette alternative, Augustin dépasse du même coup la doctrine aristotélicienne de la prédication. La relation trinitaire, en tant que relation éternelle, n'est ni substantielle, ni accidentelle : elle est au-delà de la simple attribution.

Ce dépassement de la logique attributive n'est cependant pas encore un dépassement de la pensée d'Aristote. Car plus profondément qu'une logique de l'attribution, qui n'en est que l'interprétation hellénistique tardive, le catégorial a son origine dans l'apophantique. Le dépassement du catégorial impliquerait donc un dépassement de cette dimension essentiellement monstrative qu'Aristote assigne à la parole. Or c'est bien ce que permet la parole qui tente de nommer la Trinité.

Dans la pensée d'Aristote, la relation ne peut jamais s'établir qu'entre deux ỏvoíal. La première définition qu'en donne Aristote au chapitre VII des Catégories, en 6a36, s'énonce ainsi :

«Sont dites en relation les choses telles qu'elles sont précisément dites être à d'autres. »

L'être d'une chose en relation tient ainsi à l'être de ce avec quoi elle est en relation. Mais tout relatif a-t-il, justement, un être ? Prenons l'exemple que donne Aristote. L'aile d'un oiseau est relative à l'animal ailé dont elle est l'aile. Mais une aile n'est pas, à proprement parler, un 
être. C'est ce problème qui conduit Aristote à corriger sa définition, et à dire, en 8 a 33 :

«Les choses relatives sont précisément celles pour lesquelles être est la même chose qu'avoir une relation quelconque avec quelque chose. »

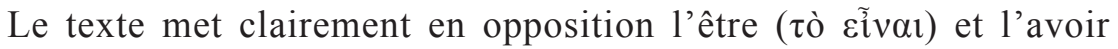

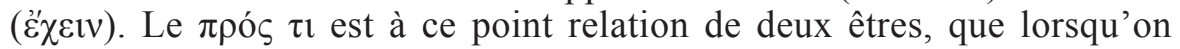
n'aura affaire qu'à un simple avoir (par exemple : avoir un corps ailé), on pensera cet avoir comme un être à part entière. Autrement dit, même si ce ne sont pas toujours des étants à part entière qui sont en relation, la catégorie de la relation exige que l'on fasse comme si on avait affaire à deux étants à part entière, c'est-à-dire à deux óvoíat.

Telle est la condition apophantique du $\pi \rho o ́ \varsigma \tau \imath$ aristotélicien. Pour que

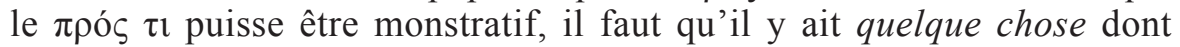
il soit la monstration. Or ce quelque chose, dont la monstration est dite essentiellement selon lui, ou est accidentellement dans lui, c'est toujours

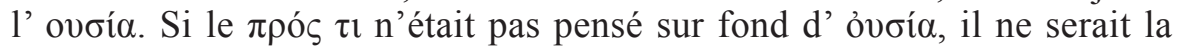
monstration de rien. L' ỏvoía est ainsi toujours et nécessairement ce à partir de quoi le $\pi \rho o ́ s \tau \imath$ peut se déployer comme monstration.

C'est à ce point que saint Augustin peut opérer un dépassement de l'apophantique. La relation trinitaire est en effet pensée en-dehors de l'alternative « substance - accident ». La relation trinitaire n'est donc, à proprement parler, relation de rien - dans un sens du mot rien qui reste à préciser. En V, IX, 10, saint Augustin écrit :

«Cependant, quand on demande : "Trois quoi ?", les ressources verbales humaines souffrent d'une grande indigence. Quoi qu'il en soit, on répond : "trois personnes", non pas tant pour dire précisément cela que pour n'être pas réduit au silence. $»^{37}$

Les « trois » dont il ici question sont ceux dont il y a, à l'intérieur de la Trinité, relation. Le terme personae, nous dit saint Augustin, est une réponse à la question : quid tres ? Cette question est en elle-même mal formulée. Car elle présuppose que les trois qui sont en relation sont pensables en termes de quiddité. Or tout quid a pour corrélat un aliquid. On se trouve ainsi contraint de parler des relations trinitaires comme s'il s'agissait de choses. Faute de mieux, on répond tres personae - le terme persona permettant de tirer le quid vers un quis, c'est-à-dire le quoi vers un qui. Mais la meilleure réponse, nous dit saint Augustin, serait le silence. A quoi ce silence tient-il ?

${ }^{37}$ De Trinitate, V, IX, 10. PL, p. 918 ; trad. fr. p. 415. 
La relation trinitaire n'est pas relation de deux êtres, mais d'un seul être. Non pas qu'elle soit relation d'un être avec lui-même. Elle est relation, en un seul être, de plusieurs : par exemple du Père et du Fils, qui sont un seul Dieu. Par conséquent, la relation n'est plus soutenue par l' óvoía. Au contraire, c'est l' ỏvoía qui est intérieurement traversée par des relations. Il faudrait donc dire que les relations trinitaires ne sont, en tant que telles, relations de rien - si l'on entend par là : rien qui relève de l' ỏvoía. Parce que le trinitaire est relation, en une même ỏvoía, de plusieurs, ces plusieurs ne sont pas des voíal. Bien entendu, les trois qui sont en relation sont tous

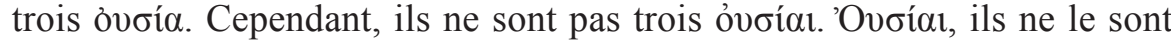
qu'en dehors de la relation, comme o ỏvбía. C'est pourquoi Augustin peut dire que sur ce point, "les ressources verbales humaines souffrent d'une grande indigence ». Cette indigence est constitutive du trinitaire lui-même. Car ce en quoi la parole humaine puise ordinairement ses ressources, c'est l' ỏvoía. Or celle-ci fait défaut à la relation trinitaire.

La relation trinitaire opère ainsi, ipso facto, une négation de l' ỏvoía. Cette négation ne se fait pas sur le modèle de la théologie négative. Augustin ne nie pas d'abord 1' óvoía pour poser ensuite la relation. Il ne dit pas de la Trinité qu'elle est une relation sans ỏvoía, comme il disait de Dieu qu'il est bon sans qualité ou grand sans quantité. La relation trinitaire travaille pour ainsi dire l' ỏvoía de l'intérieur. N'étant plus relation

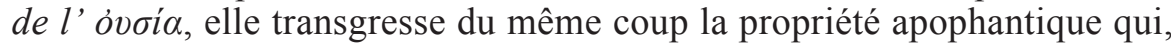
dans le $\pi \rho o ́ s \tau$ aristotélicien, lui donnait sens et mesure.

La parole qui tente de nommer la Trinité n'est donc pas, à proprement parler, une parole négative. Elle est plus profondément la négation de cela même qui, ordinairement, permet la parole. En elle, l' ỏvoía est d'emblée, c'est-à-dire à titre de condition de possibilité, niée. Plus encore qu'une parole apophatique, elle est peut-être la découverte d'une propriété apophatique de la parole en tant que telle. C'est par et dans cette propriété apophatique que l'apophantique aristotélicien peut être dépassé. Si la parole de la relation trinitaire montre quelque chose, c'est le dépassement de toute monstration. Cela devrait mener à l'étude de l'écriture même d'une telle parole. Mais ce dernier point ferait l'objet d'une étude à part entière. Nous ne pouvons ici que l'indiquer, en citant l'un des passages où cette parole, abandonnant la logique de l'ỏvoía, devient vertige logique, ou logique du vertige. Le passage se situe en X, XI, 18 :

« C'est pourquoi, lorsqu'elles sont chacune, toutes et tout entières réciproquement contenues en chacune, elles sont chacune tout entières égales à chacune tout entière, chacune tout entière est égale à elles toutes ensemble tout entières, et «ces trois sont un» $[\ldots] . »^{38}$

${ }^{38}$ De Trinitate, X, XI, 18. PL p. 984 ; trad. fr. p. 531. 
Pour conclure, disons que, au-delà de la question sans doute historiquement indécidable de savoir si Augustin a réellement lu le texte aristotélicien des Catégories, la critique augustinienne des catégories consiste à mettre en question, dans un premier temps, une logique de l'attribution pensée à partir de la notion néoplatonicienne de participation. Dieu ne participant pas à ce qu'il est, il est nécessairement supracatégorial. Mais la critique des catégories est également une critique de la substance, elle-même comprise à partir de la logique stoïcienne du substrat corporel. Dieu n'étant pas un corps, la notion de substance ne saurait lui être proprement attribuée.

Cette double caractérisation des catégories, nous avons tenté de la mesurer au catégorial aristotélicien. L' ỏvoía, qui en constitue la dimension principale, ne saurait être pensée à partir d'une logique du substrat, puisqu'elle se déploie à partir d'un déictique qui, loin de se limiter aux corps, manifeste la présence simple d'un « ceci que voici » ( $\tau$ ó $\delta \varepsilon \tau \imath)$, présence qui se prolonge dans sa propre dimension de visibilité - autrement

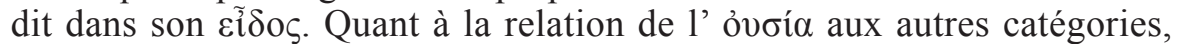
elle n'est évidemment pas déterminée par Aristote comme participation, mais comme rapport apophantique - rapport que nous avons interprété comme monstration et manifestation.

Mais si la critique augustinienne des catégories n'atteint pas directement la lettre du texte aristotélicien, elle ne permet pas moins un dépassement du catégorial comme tel, c'est-à-dire de l'apophantique qui en constitue le fond. L'ineffable se substitue à la monstration. On pourrait alors s'attendre à la simple substitution d'une parole apophatique à la parole apophantique. Mais plus encore qu'une parole apophatique, la parole de la relation est la découverte d'une propriété apophatique de la parole en tant que telle. Parce qu'elle est relation, en une même ỏvoía, de plusieurs, ces plusieurs ne sont pas des ỏvoíal. La relation n'est plus relation de quelque chose, mais relation, à proprement parler, de rien c'est-à-dire de rien qui soit de l'ordre de l' óvoía. La relation n'est plus la monstration de ce dont elle est la relation. Elle est la monstration du dépassement de la monstration.

Tel nous semble être du moins l'acquis de la critique augustinienne des catégories d'Aristote, dès lors qu'elle n'est plus simplement confrontée à l'aristotélisme, mais bien à la pensée d'Aristote. Plus que la substitution d'une logique à une autre, elle permet la découverte d'une nouvelle possibilité de la parole. Parole du dépassement de la parole, c'est-à-dire de l'ineffable qui, singulièrement, continue de se découvrir par et dans la parole. C'est ce que permettrait peut-être de confirmer une étude de l'écriture de la relation dans le De Trinitate. 